\title{
La leishmaniasis: conocimientos y prácticas en poblaciones de la costa del Pacífico de Colombia
}

\author{
Diana María Isaza, ${ }^{1}$ Berta Nelly Restrepo, Margarita Arboleda, ${ }^{1}$ \\ Eudoro Casas, ${ }^{1}$ Herminio Hinestroza ${ }^{2}$ y Tufik Yurgaqui ${ }^{2}$
}

RESUMEN En 1997 se realizó un estudio descriptivo con énfasis cualitativo con objeto de documentar los conocimientos y las prácticas relacionados con la leishmaniasis cutánea, según el sexo, en los habitantes de 14 años en adelante de siete comunidades del departamento del Chocó, Colombia. Los residentes de esa zona de la costa del Pacífico estaban en alto riesgo de contraer leishmaniasis, que se conoce localmente con los nombres de bejuco y yateví, ya que las actividades de control llevadas a cabo por el Servicio Seccional de Salud del Chocó no habian tenido el éxito deseado. Se recogieron datos cualitativos involucrando directamente a cada comunidad en talleres de discusión y entrevistando a informantes calificados. Sobre esa base, se elaboró una encuesta de 10 preguntas cerradas, que los investigadores administraron a todas las personas mayores de 14 años presentes en cada hogar elegido de forma aleatoria para ser visitado. Los resultados indican que $94 \%$ de la población estudiada conocía la leishmaniasis como una enfermedad de la piel y este concepto era más común entre los hombres que entre las mujeres. En cuanto a la forma de transmisión, 35\% relacionaron la enfermedad con la picadura de un insecto, pero ignoraban el agente etiológico y consideraban que la producía un gusano que vive en el monte. En las comunidades estudiadas se empleaba una gran variedad de tratamientos para curar la enfermedad. Estos se basaban en el uso de plantas, sustancias químicas, quemadura de las lesiones con metales calientes y, en menor proporción, medicamentos. A pesar de que las mujeres tenían la responsabilidad de cuidar a los enfermos en el hogar, su falta de conocimientos sobre el tratamiento fue una variable de análisis estadísticamente significativa, pues no estaban familiarizadas con los tratamientos tradicionales de su comunidad. De las personas encuestadas, 45\% no sabian cómo prevenir la enfermedad, especialmente las mujeres, según pudo observarse en 102 de 155 respuestas. No se encontraron diferencias atribuibles al género en los conocimientos y prácticas acerca de la leishmaniasis, con excepción de la falta de conocimiento de las mujeres acerca del tratamiento de la enfermedad. Esta investigación resalta la importancia de estudiar los conocimientos y prácticas de los habitantes locales antes de diseñar y organizar programas educativos sobre el control de la leishmaniasis.

1 Instituto Colombiano de Medicina Tropical, Medellín, Colombia. Toda correspondencia referente a este artículo deberá enviarse a Diana María Isaza, a la siguiente dirección: Cra. 51 A No. 62-42, Medellín, Colombia, A.A. 52162. Correo electrónico: icmt@epm.net.co

2 Servicio Seccional de Salud del Chocó, Quibdó, Colombia.
La leishmaniasis es una zoonosis de amplia distribución mundial que representa un problema de salud pública en muchos países del Tercer Mundo. En Colombia, la enfermedad es endémica y se encuentra en $91 \%$ de todo el territorio ubicado bajo los $1750 \mathrm{~m}$ sobre el nivel del mar. Durante 1995, se registraron casos de la enfermedad en $45 \%$ de los municipios del país. La leishmaniasis es una dolencia crónica, lo que hace difícil distinguir 
los casos nuevos. Por lo tanto, los datos aportados por el Ministerio de Salud se presentan en proporciones de prevalencia, con la población rural de los municipios endémicos como denominador. Durante el período de 1985 a 1996, se informó de 55888 casos de leishmaniasis, entre los que predominó la forma cutánea (95\%). Se registraron prevalencias que oscilaron entre 18,03 y 57,63 , con una media de 61,1 por 100000 habitantes (1).

La costa de Colombia sobre el Pacífico, donde se realizó la investigación que relatamos, está dividida en cuatro departamentos, de los cuales el del Chocó ocupa el territorio más extenso. Es una zona de bosque húmedo tropical donde cae una cantidad de lluvia mayor que en todo el resto del continente americano y la temperatura varía entre los 23 y $32{ }^{\circ} \mathrm{C}$. La humedad ambiental es de 90\%, aproximadamente (2). Según datos de 1997 del Ministerio de Salud, el Chocó es un área de alto riesgo de leishmaniasis y tiene una tasa ajustada de 243,73 casos por 100000 habitantes (1).

Como parte de la búsqueda activa de casos de leishmaniasis, el Servicio Seccional de Salud del Chocó ha preparado carteles y anuncios sobre la enfermedad que se distribuyen en los hospitales y puestos de salud. Estos últimos están a cargo de auxiliares de enfermería que, además de otras tareas, remiten a los pacientes cuyos cuadros clínicos son compatibles con la leishmaniasis a las unidades hospitalarias para el diagnóstico. También administran el tratamiento con Glucantime $^{\circledR}$ a los pacientes que lo requieran. Dichas auxiliares son residentes de las comunidades locales, lo que garantiza su estabilidad en el trabajo. Sin embargo, se ha observado que esas actividades no han tenido el efecto deseado en las prácticas de la comunidad para prevenir y manejar la enfermedad. Por otro lado, no se han realizado muchos estudios que recojan los conceptos populares sobre la leishmaniasis y sirvan para crear programas de educación para los habitantes de las zonas endémicas con respecto al control de la enfermedad en consonancia con las prácticas de la población.
Dadas las circunstancias epidemiológicas y logísticas descritas, y el hecho de que en la costa del Pacífico del Chocó habita un grupo étnico diferente de los demás que pueblan el país (3-4), se vio la necesidad de realizar el presente estudio durante 1997. El objetivo principal fue describir los conocimientos populares acerca de la presentación clínica y el modo de transmisión de la leishmaniasis, así como los tratamientos y las formas de prevención que practicaban los habitantes de esa zona. Se prestó atención especial a las diferencias por género.

\section{MATERIALES Y MÉTODOS}

De 17 comunidades habitadas por poblaciones de raza negra en los municipios de Bahía Solano y Nuquí, se seleccionaron para el estudio siete colectividades rurales con prevalencias similares de leishmaniasis, tres en Bahía Solano (Cupica, Nabugá y El Valle) y cuatro en Nuquí (Tribugá, Panguí, Coquí y Arusí). Durante el período de 1989 a 1995 se registraron 52 casos en Nuquí y 53 en Bahía Solano. Esos municipios costeros están situados dentro de una faja de 150 km del litoral Pacífico. Los criterios de selección se basaron en los registros de investigaciones anteriores que mostraban casos confirmados de leishmaniasis en cada una de esas comunidades. ${ }^{3}$ Además, se seleccionaron poblaciones de más de 250 habitantes que fueran razonablemente accesibles y donde se pudiera contar con la presencia de promotores del Centro Internacional de Educación y Desarrollo Humano (CINDE) para que contribuyeran a promover la investigación entre la comunidad.

\section{Recolección de datos cualitativos}

Como estrategia para la recolección de datos cualitativos se llevaron a cabo

\footnotetext{
3 Corporación para Investigaciones BiológicasPatrulla Aérea Colombiana. Prevalencia de leishmaniasis cutánea y mucocutánea a lo largo de 200 kilómetros de costa Pacífica Chocoana, 1986; Brigadas de Salud, Patrulla Aérea Colombiana, Seccional Antioquia. Registros de consulta externa, 1986-1994.
}

dos talleres de discusión con miembros y grupos focales de cada comunidad. Los investigadores fueron de casa en casa invitando a los habitantes a asistir a los talleres y citaron, además, a grupos focales como los de maestros y promotores de la salud. En los talleres, los asistentes tuvieron la oportunidad de exponer sus conocimientos y opiniones sobre el modo de transmisión de la leishmaniasis, de acuerdo con las preguntas preparadas por los investigadores. Ello facilitó la discusión y el intercambio de información entre los miembros de la comunidad acerca de las prácticas de prevención y tratamiento acostumbradas.

Los investigadores también entrevistaron a informantes calificados, es decir, a aquellas personas que habían estado en mayor contacto con la enfermedad, como los promotores de salud, los curanderos encargados de tratarla y los propios pacientes de leishmaniasis, así como sus familiares y convivientes. Para estas entrevistas, que fueron de tipo estructurado, se empleó una guía (5). Además, se obtuvo previamente el consentimiento verbal de los entrevistados para grabarlas en audiocasetes. Las entrevistas tuvieron dos enfoques: los conocimientos y las prácticas sobre la leishmaniasis y los papeles que desempeña cada uno de los sexos frente a la situación de enfermedad. Las grabaciones fueron posteriormente transcritas y de ellas se sacó parte de la información para el diseño de encuestas destinadas a obtener datos cuantitativos.

Toda la información cualitativa se categorizó por variables principales según las respuestas obtenidas en las siete comunidades. A continuación se verificó la validez de los datos siguiendo métodos establecidos (5). La triangulación de datos se llevó a cabo después de haber recolectado toda la información, durante el proceso de su interpretación

\section{Recolección de datos cuantitativos}

Sobre la base en la información obtenida durante las discusiones de grupo y las entrevistas de informantes calificados, se diseñó una encuesta corta con 
FIGURA 1. Distribución de la población encuestada por sexo y edad, Chocó, Colombia, 1997

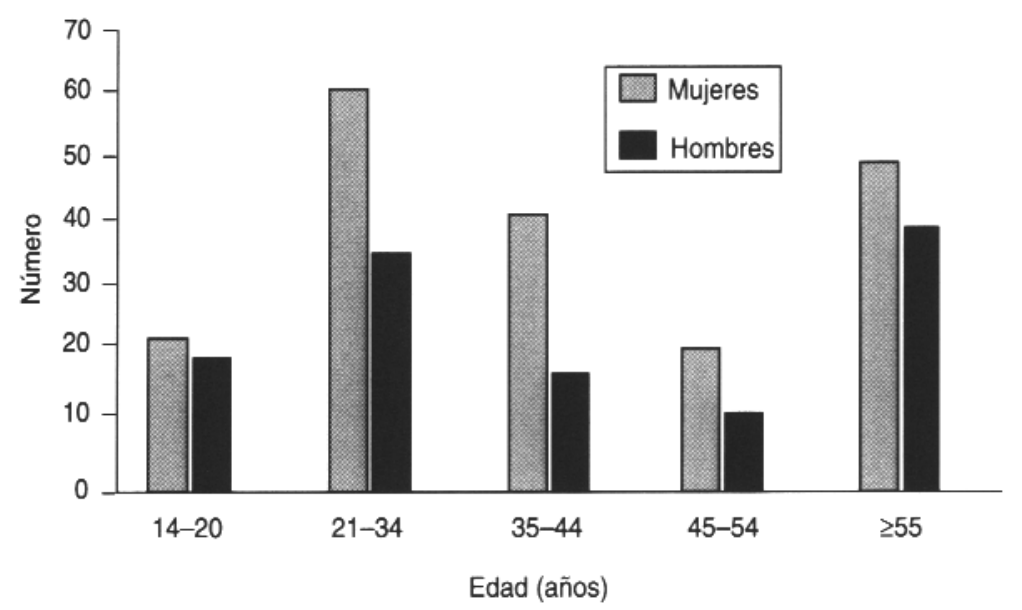

FIGURA 2. Forma de transmisión de la leishmaniasis según la población encuestada, Chocó, Colombia, 1997

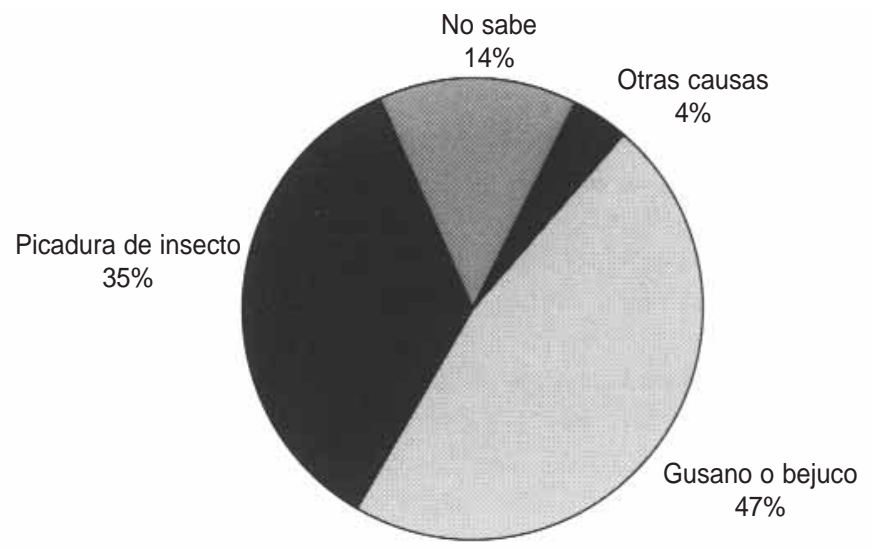

10 preguntas cerradas sobre los conocimientos y prácticas acerca de la leishmaniasis y los papeles que desempeñan hombres y mujeres frente a la situación de enfermedad. Se utilizaron como opciones las mismas respuestas que se validaron en el análisis cualitativo (anexo 1).

El número total de encuestados se calculó sobre la base de la población mayor de 14 años residente en las siete comunidades ( $n=1553)$, con un error muestral de $10 \%$, una prevalencia estimada de $50 \%$ y un nivel de confianza de $95 \%$, lo que dio un tamaño muestral de 340 encuestados teniendo en cuenta una pérdida de $10 \%$. La distribución representativa del número de encuestas por comunidad se basó en el porcentaje que ocupaban los mayores de 14 años de cada comunidad con respecto al total de la población.

Las casas encuestadas se seleccionaron mediante un muestreo sistemático de una de cada cuatro o seis viviendas, dependiendo del número en cada comunidad y del número de encuestas por realizar, suponiendo tres entrevistas por hogar. Las encuestas fueron administradas por los investigadores, previamente asesorados por expertos del Centro Internacional de Educación y Desarrollo Humano, acompañados de líderes comunitarios. Se encuestó a todos los mayores de 14 años presentes en la vivienda en el momento de visitarla. Antes de comenzar la encuesta se le explicó a cada individuo el motivo de la investigación y se tomó su consentimiento informado por escrito. Se trató de encuestar a cada individuo a solas, especialmente a los más jóvenes, para evitar que los mayores respondieran por ellos. El total de encuestados ascendió a 135 varones y 210 mujeres.

Tanto el cálculo de la muestra como el análisis estadístico de las encuestas se hicieron con el programa Epi Info 6 (6). La prueba de significación estadística de ji al cuadrado $\left(x^{2}\right)$ se aplicó al análisis de las respuestas sobre conocimientos y prácticas por sexo.

\section{RESULTADOS}

Se encuestó en total a 345 personas, de las cuales $61 \%$ eran mujeres y $39 \%$, hombres. La figura 1 muestra la distribución por sexo y edad de la población encuestada, que se componía totalmente de personas de raza negra.

En la región estudiada, la leishmaniasis se conoce con los nombres de bejuco y yateví. Solo 6,7\% de los encuestados conocían la enfermedad con el nombre de leishmaniasis. Este resultado fue semejante en ambos sexos y en todos los grupos de edad. En $47 \%$ prevalecía la idea de que las personas sufren de leishmaniasis porque van al monte y allí les pica un gusano que se encuentra en el barro o en las hojas de los árboles o porque son tocados por un bejuco o liana. Una proporción menor $(35 \%)$ relató que la enfermedad se adquiere mediante la picadura de un mosquito infectado, es decir, se asoció con la idea de que el mosquito posee algo que produce la enfermedad. Entre $4 \%$ de los encuestados, el origen de la enfermedad se atribuyó a otras causas, por ejemplo, heridas accidentales, contagio de personas enfermas o falta de aseo, mientras que $14 \%$ respondió que no sabía cómo se transmite la leishmaniasis (figura 2). No hubo diferencias significativas entre los conceptos de hombres y mujeres. En el grupo de edad de 21 a 34 años se encontró el mayor porcentaje de personas $(37 \%)$ 
FIGURA 3. Conocimientos de la población encuestada, según el sexo, acerca de la presentación clínica de la leishmaniasis, Chocó, Colombia, 1997

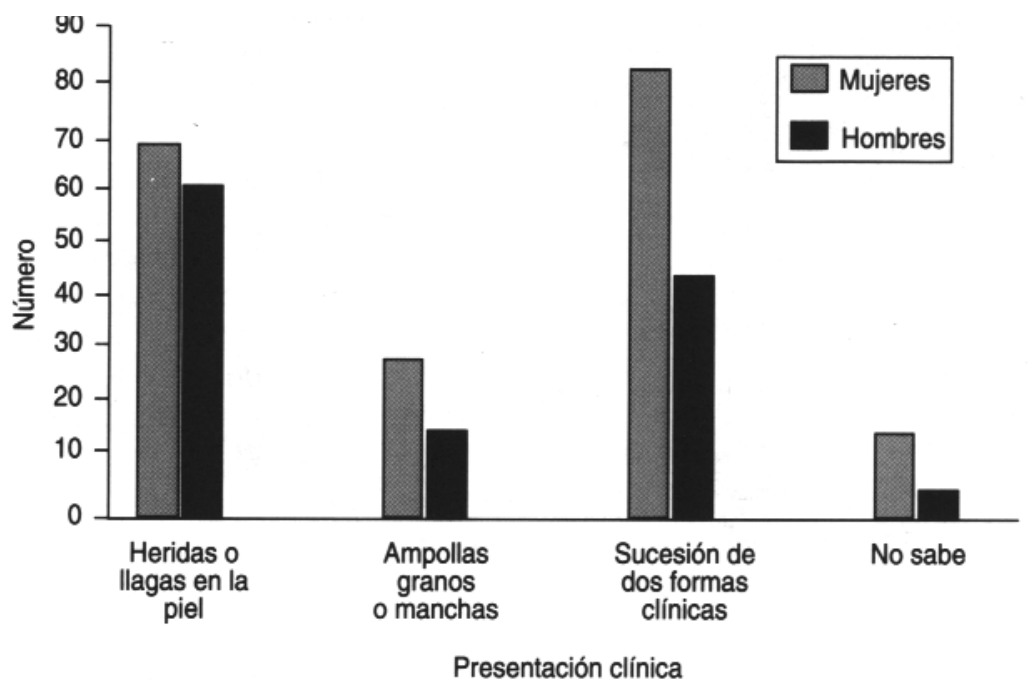

que conocían la forma de transmisión por picadura de insecto.

En cuanto a la presentación clínica de la enfermedad, $94 \%$ de las personas encuestadas conocían el bejuco como una enfermedad de la piel, que puede presentarse en forma de úlceras $(40 \%)$, ampollas, granos o manchas $(15 \%)$, o la sucesión de dos de esas formas clíni- cas $(39 \%)$, y relacionaron la presencia de prurito (rasquiña) con la aparición de una "ampolla o grano, que al poco tiempo se revienta como resultado de la rasquiña y comienza a crecer en forma redonda". Ellos reconocían que las úlceras se localizan frecuentemente en las extremidades y con menor frecuencia en la cara y la espalda. El co-

FIGURA 4. Conocimientos y prácticas acerca del tratamiento de la leishmaniasis, según el sexo, Chocó, Colombia, 1997

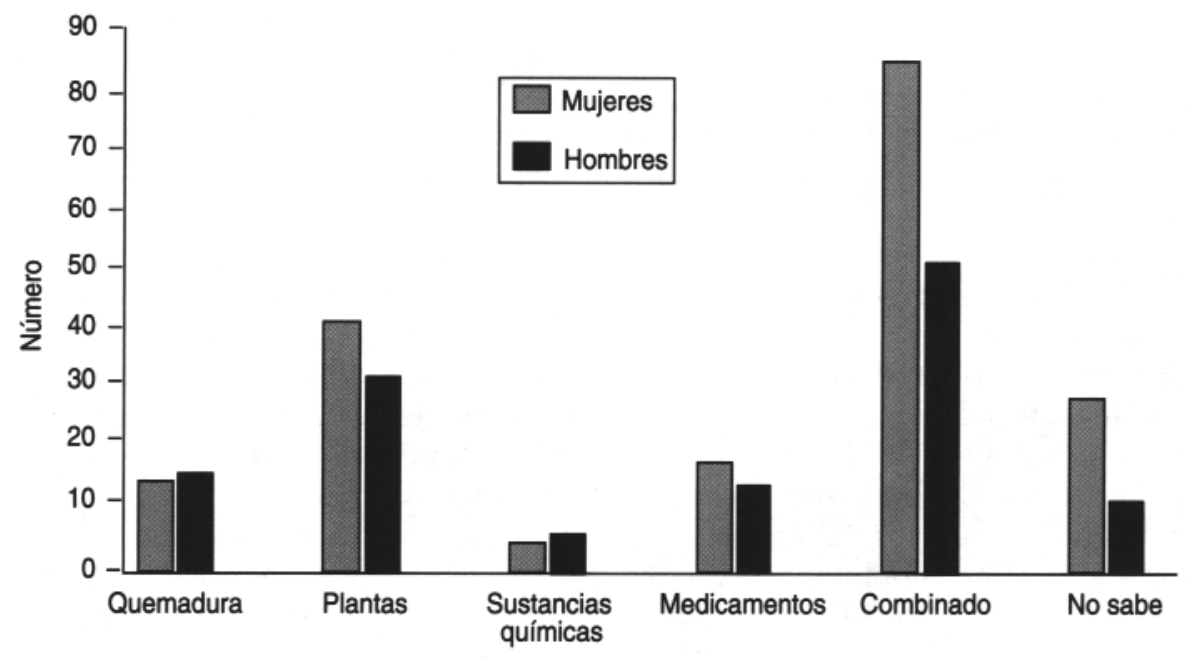

Tipo de tratamiento

nocimiento de la enfermedad como úlceras en la piel fue muy similar en ambos sexos. De $6 \%$ que dijeron que no sabían cómo se presentaba la enfermedad, hubo una proporción más alta de mujeres que de hombres $(77,3 \%$ frente a $22,7 \%$ ) pero esta diferencia no fue estadísticamente significativa (figura 3).

El tratamiento de la enfermedad se basaba en el uso de las raíces, hojas o frutos de ciertas plantas, en forma de emplastos o bebidas o en el lavado de las lesiones (23\%). Otra práctica generalizada consistía en quemar la lesión con una cuchara de plata caliente o algún otro instrumento de metal $(10 \%)$, o usar sustancias químicas como ácido de batería, ácido sulfúrico, ácido bórico, hipoclorito de sodio, yodo, ácido salicílico, timerosal, cresol, alcohol o petróleo $(4 \%)$. Algunas $(41 \%)$ de las personas encuestadas combinaban varios tratamientos. Los medicamentos (incluidas las inyecciones) se usaban poco para curar la leishmaniasis $(10 \%)$ y en las entrevistas solo las mencionaron las personas que habían sufrido la enfermedad y finalmente fueron tratadas con Glucantime ${ }^{\circledR}$ en el hospital local. Tanto hombres como mujeres utilizaban las plantas como primera medida de tratamiento, seguidas de las quemaduras con metal caliente $\mathrm{y}$, en tercer lugar, de las inyecciones. Al comparar las respuestas según el sexo se observó que una mayor proporción de hombres preferían las medidas que cauterizan la lesión (quemadura con metal caliente y aplicación de sustancias químicas) y las mujeres, el uso de plantas y antibióticos.

Se presentó una diferencia estadísticamente significativa por sexo en cuanto al número de encuestados que no conocían ningún tratamiento para la leishmaniasis, que fue mayor en mujeres que en hombres (RR: 2,22; IC95\%: 1,09 a 4,52) (figura 4). El uso o el conocimiento de tratamientos tradicionales como la cauterización, las plantas y las sustancias químicas fue más frecuente en el grupo de edad de más de 55 años. En el de de 21 a 34 años fue más frecuente reconocer que no sabían cómo se trata la enfermedad. En todas las comunidades, con excepción de Coquí, las plantas fueron la 
primera elección para el tratamiento de la leishmaniasis. La cauterización ocupó los lugares segundo y tercero.

Las personas que presentaban lesiones compatibles con la leishmaniasis consultaban al curandero o al médico de hierbas (20\%) porque creían en el poder de las plantas para curar enfermedades, especialmente la leishmaniasis. No obstante, sabían que es una enfermedad que pueden curar los médicos $(27 \%)$, pero los hospitales locales estaban muy lejos y el transporte marítimo era muy costoso. También era frecuente consultar con los promotores de salud $(17 \%)$, pero ellos solamente podían desinfectar las lesiones porque ignoraban cómo tomar las muestras para el diagnóstico. En una menor proporción, se consultaba a las personas mayores de edad de la comunidad (6\%), debido a que tenían mucha más experiencia y conocían muy bien la enfermedad. Asimismo, 23\% de las personas encuestadas consultaban a dos o más personas, sobre todo al curandero y al médico (10\%). La consulta con el médico o con personas mayores de edad era más común en las mujeres (61,5\% y 63,2\% respectivamente) que en los hombres, pero esta diferencia no fue estadísticamente significativa. La consulta con curanderos y promotores de salud fue muy similar en ambos sexos. El grupo de edad de más de 55 años prefería consultar con el curandero o personas mayores de edad, mientras que el grupo de 21 a 34 años prefería al médico o al promotor o promotora de salud.

Los que pensaban que la enfermedad se adquiere por contacto con un animal o con un mosquito dijeron que la forma más fácil de prevenirla es cubrirse bien el cuerpo antes de ir al monte $(24 \%)$ y usar mosquiteros en las casas (2\%). Entre las formas de prevención mencionaron también gritar "ya te vi" al entrar en el monte $(7 \%)$, pues de esta manera el gusano ya no pica. Otras formas de prevención, mencionadas por $20 \%$ de la población encuestada fueron cortar el bejuco, andar con cuidado en el monte, cuidar los pozos sépticos, hacer desmonte, fumigar, no ir al monte, no acercarse a nadie que tenga bejuco e ingerir líquidos en
FIGURA 5. Conocimientos, según el género, acerca de medidas para prevenir la leishmaniasis, Chocó, Colombia, 1997

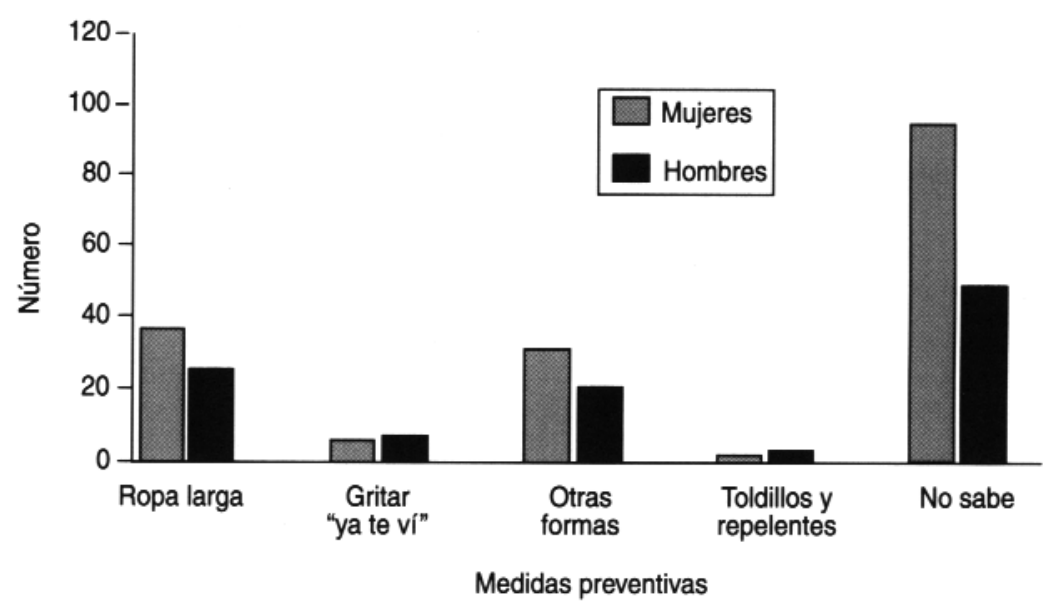

abundancia para que muera el bejuco. Un número considerable expresó que la enfermedad "no se puede evitar", "es muy difícil de evitar" o no sabían la manera de prevenirla (47\%). Más mujeres que hombres declararon que desconocían la forma de prevenir el bejuco $(65,8 \%$ frente a $34,2 \%)$, pero esta diferencia no fue estadísticamente significativa (figura 5). En las respuestas a la pregunta sobre prevención no hubo diferencias relacionadas con la edad. La única medida de prevención mencionada en las siete comunidades fue la de usar ropa que pueda cubrir bien el cuerpo.

En lo que se refiere a los papeles que desempeñaban los dos sexos frente a la situación de enfermedad, $42 \%$ de los encuestados respondieron que quien se encarga de buscar atención es la madre y $29 \%$, el padre. Las mujeres tendieron a mencionar a la madre y los hombres al padre como persona responsable de buscar atención. Las personas encuestadas menores de 44 años consideraban a la madre responsable de buscar atención, mientras que los mayores de 45 años señalaron al padre. Asimismo, 81,4\% respondieron que la madre es la encargada de cuidar al enfermo; $8,1 \%$ que el padre lo hace, $y$ un porcentaje menor mencionó a los abuelos o los hermanos mayores. Tanto hombres como mujeres reconocían que la madre es la responsable principal del cuidado del enfermo y ese re- sultado coincidió en todos los grupos de edad.

\section{DISCUSIÓN}

La leishmaniasis cutánea es una enfermedad que se encuentra ampliamente distribuida a lo largo de la costa colombiana del Pacífico (7). La prevalencia de la enfermedad en las siete comunidades estudiadas era similar y en la mayoría de ellas se consideraba una de las enfermedades endémicas de atención prioritaria.

A pesar de que en las comunidades estudiadas la distribución de hombres y mujeres era similar, con un ligero predominio del sexo masculino, la representatividad del mismo en la población de encuestados se vio afectada porque las encuestas se hicieron durante el día. Se entrevistó a un número mayor de mujeres, ya que los hombres de edad productiva se encontraban en el monte trabajando o pescando. $\mathrm{La}$ influencia del factor de la hora se reflejó también en el sexo de los asistentes a los talleres de discusión, con una mayor asistencia de hombres en los talleres nocturnos y menor en los talleres diurnos. Por otra parte, esto también indica que en las horas de la noche las mujeres preparaban los alimentos o se quedaban a cargo de los niños mientras los hombres asistían a la reunión. 
No se cuantificó el número de habitantes que rehusaron participar en la encuesta, pero se trató de muy pocas personas. En esos pocos casos, la razón fue el temor de firmar para dar su consentimiento, a pesar de que verbalmente habían aceptado responder a la encuesta.

El nombre de bejuco o yateví para designar la leishmaniasis tiene que ver con las creencias de la población respecto de la forma como se adquiere la enfermedad. El uso de estos nombres fue descrito también por Vásquez et al. (8) en relación con otras poblaciones al sur del Chocó situadas, aproximadamente, a $300 \mathrm{~km}$ de Cupica. Llama también la atención que, en un estudio similar realizado en el Ecuador en la provincia de Pichincha, a $100 \mathrm{~km}$ de la costa del Pacífico, Weigel et al. (9) encontraron que la leishmaniasis cutánea era llamada la colombiana debido a que las úlceras y cicatrices eran marcas características de quienes venían de zonas endémicas de la costa colombiana del Pacífico.

Si bien algunos habitantes de las siete comunidades estudiadas saben que la leishmaniasis puede ser transmitida por mosquitos, la mayoría creen que es transmitida por la picadura de un gusano o el contacto con un bejuco. Vásquez et al. (8) informan de la misma creencia en $45 \%$ de la población que estudiaron, porcentaje similar al nuestro. Sin embargo, en ese estudio no se implica ninguna planta particular en la transmisión de la enfermedad, a diferencia del nuestro, en el que algunos participantes describieron la planta como "un bejuco que echa una flor amarilla" o "es un árbol grande; el palo echa una resina y esa resina es la que se trae". Ambos conceptos reflejan la connotación especial de que la leishmaniasis es una enfermedad exclusivamente del monte, frente a la cual los habitantes sienten cierta impotencia para prevenirla. Además, consideran que los hombres están en mayor riesgo de contraer la enfermedad que las mujeres, lo que se debe probablemente a la diferencia de exposición ocupacional entre ambos sexos. Estos hallazgos muestran un desconocimiento de la forma de trans- misión de la enfermedad que podría afectar a la ejecución de programas de control.

En las comunidades estudiadas prevalecían los tratamientos tradicionales mediante el uso de plantas, quemaduras con metales calientes y sustancias químicas, tal cual informan también otros autores $(8,9)$. Los hombres expresaron preferencia por la quemadura con metales calientes y sustancias químicas y, en un estudio similar, esos tratamientos se denominaron fuertes, ya que ulceran la lesión como consecuencia directa de su aplicación (8). El uso de ácido de batería también ha sido descrito por otros investigadores en la costa de Colombia sobre el Pacífico, en los departamentos de Nariño y Valle (10), y en Costa Rica (11). Las mujeres prefieren el uso de plantas y antibióticos, tratamientos denominados suaves o menos fuertes (8). Esto muestra la tendencia del hombre a acudir a recibir tratamientos más agresivos, dada la creencia cultural en el mayor umbral de dolor en el hombre.

No se encontraron diferencias en cuanto a la oportunidad de acceso de un sexo $u$ otro a recibir alguna clase de tratamiento. Lo mismo se ha comentado de otra población (9). Además, se encontró que las mujeres preferían consultar con un médico, lo que muestra que ambos sexos tienen igual acceso a los escasos servicios de salud, contrario a lo encontrado en otras comunidades del interior del país (12). La significativa falta de conocimiento de las mujeres sobre el tratamiento puede explicarse por la baja incidencia de la enfermedad que se registra entre ellas en la región. Los hombres se preocupan más por la posibilidad de sufrir de leishmaniasis y es probable que conozcan mejor el tratamiento por la experiencia de otros.

Desde el punto de vista de la salud pública, llama la atención el desconocimiento general entre la población de las formas de prevenir la enfermedad, lo cual sugiere directrices sobre temas que deben tratarse en los programas de educación y control en la zona de estudio. Con respecto a quién acudir al sentirse enfermo, el análisis cualitativo indica que la mayoría acude al curan- dero; pero en las encuestas aparece como primera opción la consulta con un médico y como segunda opción el curandero, lo cual consideramos que puede ser un sesgo por la presencia de los investigadores, tres de los cuales son médicos, en el momento de la encuesta.

Se destaca la preferencia de los mayores de 55 años a consultar con el curandero, lo que muestra su preferencia por la medicina tradicional, vigente por muchos años. Los jóvenes de 21 a 34 años consultan con el médico o el promotor o la promotora, lo que refleja una nueva tendencia de los últimos años como resultado de un acceso más fácil a los servicios de salud. Sin embargo, cabe resaltar que frente a la situación de enfermedad, cada sexo defiende su desempeño frente a la responsabilidad de buscar atención para el enfermo, aunque los hombres reconocen que la aplicación de tratamientos y el cuidado del enfermo están a cargo de la mujer, pues es la que permanece más tiempo en el hogar.

Los resultados de la presente investigación muestran la importancia de los estudios de conocimientos y prácticas al diseñar y estructurar programas educativos y de intervención. Asimismo, resalta el valor de los métodos cualitativos, los cuales permiten obtener una gran cantidad de información involucrando directamente a la comunidad, en contraste con la participación pasiva que ocurre cuando los miembros de una comunidad están limitados a responder a las preguntas de una encuesta. No obstante, los métodos cuantitativos permiten validar la información, midiendo la distribución del fenómeno y la significación estadística. De esta forma, los métodos cualitativos involucran la participación de la comunidad para que conozcan su propia realidad, un paso esencial para iniciar cualquier proceso de transformación.

Cabe notar que, al evaluarse el primer taller realizado en las comunidades, los participantes expresaron su descontento porque no estaban recibiendo nuevos conocimientos de los investigadores. Ello se debió en parte a la falta de retroinformación a la co- 
munidad después de otros estudios realizados en la zona por otras instituciones. Por esa razón, y como parte del trabajo educativo que se deseaba realizar en la comunidad, se diseñó una cartilla instructiva adecuada a las condiciones de la zona. La cartilla se elaboró después de terminadas las encuestas y estaba dirigida a la población en general, incluida la población escolar. En ellas se describe de forma muy sencilla, mediante texto e ilustraciones, qué es la leishmaniasis, qué agente la produce, dónde se crían los mosquitos que la transmiten, cómo puede adquirise la enfermedad (ciclo de vida del parásito), cuándo debe sospecharse que una persona tiene leishmaniasis, cómo se trata y cómo puede prevenirse.

Asimismo, durante el taller final posterior a las encuestas, junto con las cartillas se diseñó un juego para dinamizar el proceso educativo. Dicho juego, mediante preguntas, afianzaba en los participantes los conceptos consignados en la cartilla y permitía discutir las respuestas. Ese material se entregó también a los maestros para que lo aplicaran a la población escolar.

\section{REFERENCIAS}

1. Cepeda I. Leishmaniasis en Colombia. Santa Fe de Bogotá: Ministerio de Salud de Colombia, División Técnica: Unidad Administrativa Especial de Campañas Directas (UAECD); 1997.

2. Departamentos de Colombia: Chocó. En: Periódicos asociados, ed. Nuevo Atlas de Colombia. Cali, Colombia: Prensa Moderna Impresores; 1997. pp. 184-185.

3. Peralta JA. Esclavitud y trata: un infame comercio de sangre y dolor. Periódico El Colombiano, 21 de enero de 1996, p. 6D.

4. Mosquera S, Mosquera O, Gracia JE. Geografía e historia integrada del Chocó. Caja de Previsión Social del Magisterio del Chocó. Medellín: Editorial Trama; [sin año].

5. Rodríguez Cehk P, Bonilla-Castro E. La investigación en ciencias sociales: más allá del dilema de los métodos. Santa Fe de Bogotá: Editorial CEDE; 1995.

6. Epi Info 6 [programa de computadora, versión 6.01.]. Atlanta, Georgia: Centros para el Control y la Prevención de Enfermedades
Agradecimiento. Esta investigación fue financiada por el Programa Especial de Investigaciones y Enseñanzas sobre Enfermedades Tropicales, Programa de las Naciones Unidas para el Desarrollo/Banco Mundial/Organización Mundial de la Salud, proyecto ID 960196.

Agradecemos también la inestimable colaboración de los doctores Fernando Peñaranda y Sonia Botero, del Centro Internacional de Educación y Desarrollo Humano (CINDE), por su asesoramiento y apoyo en la ejecución del proyecto. y Ginebra: Organización Mundial de la Salud; 1994.

7. Weigle KA, Saravia NG, Dávalos M, Moreno LH, D'Alessandro A. Leishmania braziliensis from the Pacific coast region of Colombia: foci of transmission, clinical spectrum and isoenzyme phenotypes. Am J Trop Med Hyg 1986; 35:722-731.

8. Vásquez ML, Kroeger A, Lipowsky R, Alzate A. Conceptos populares sobre la leishmaniasis cutánea en Colombia y su aplicabilidad en programas de control. Bol Oficina Sanit Panam 1991;110:402-415.

9. Weigel MM, Armijos RX, Racines RJ, Zurita C, Izurieta R, Herrera E, et al. La leishmaniasis cutánea en la región subtropical del Ecuador: percepciones, conocimientos y tratamientos populares. Bol Oficina Sanit Panam 1994;117: 400-412.

10. Werner JK, Barreto P. Leishmaniasis in Colombia: a review. Am J Trop Med Hyg 1981; 30:751-761.
11. Dobles-Ulloa A, Perriard C. Representaciones, actitudes y prácticas respecto a la leishmaniasis cutánea en la población del Cantón de Acosta, Provincia de San José, Costa Rica: estudio antropológico exploratorio. Cad Saude Publica 1994;10:181-189.

12. Vélez ID, Hendrickx E, Roman O, Agudelo S. Gender and leishmaniasis in Colombia: a redefinition of existing concepts. UNDP/ World Bank/WHO Special Programme for Research and Training in Tropical Diseases (TDR); 1997.

Manuscrito recibido el 26 de octubre de 1998 y aceptado para publicación, tras revisión, el 29 de junio de 1999. 
ANEXO 1. Encuesta sobre conocimientos y prácticas acerca de la leishmaniasis cutánea en comunidades del departamento del Chocó, Colombia, 1997

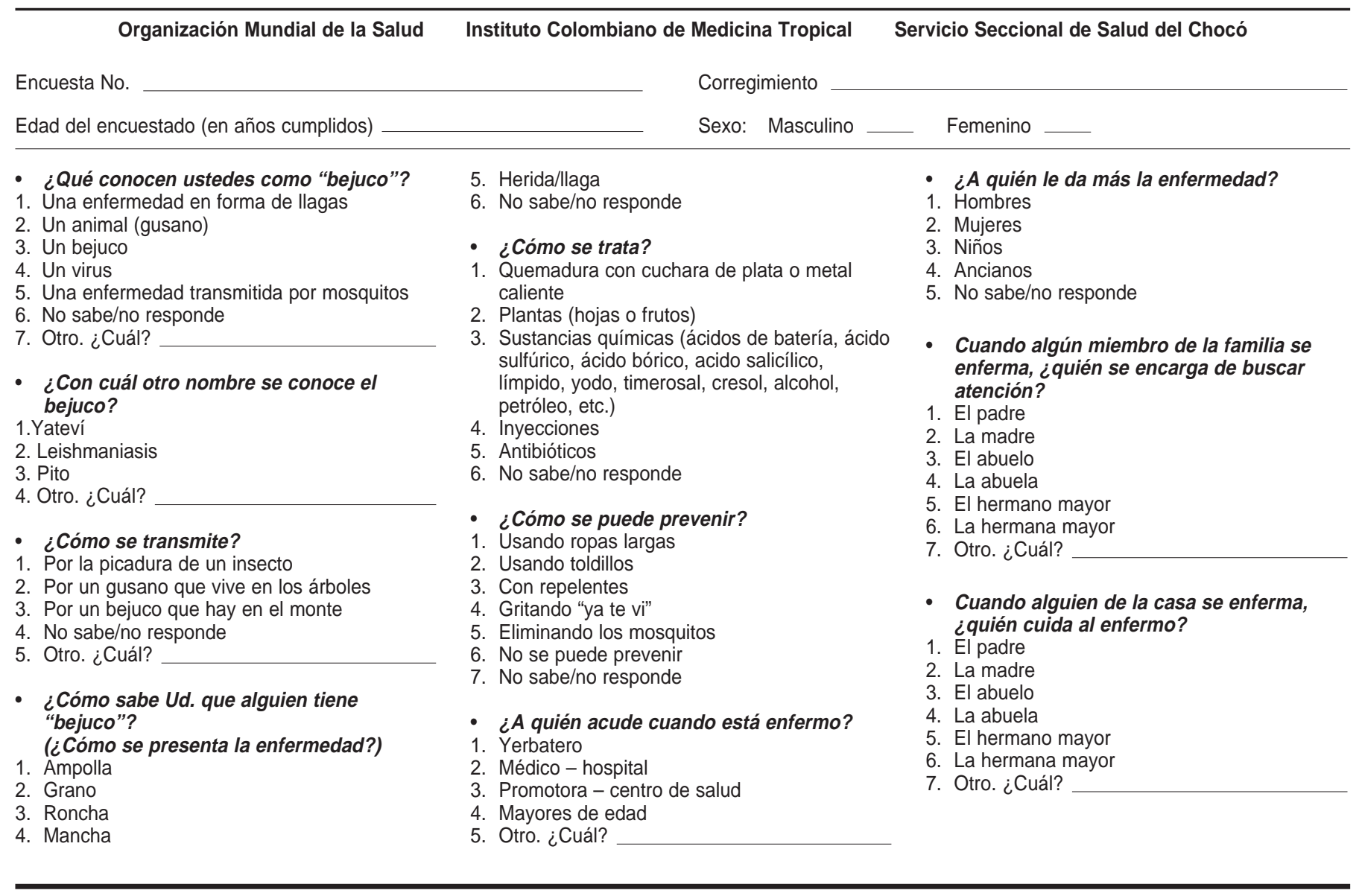

ABSTRACT In 1997 a descriptive study with a qualitative emphasis was carried out in order to document, by gender, the knowledge and practices related to cutaneous leishmaniasis among inhabitants 14 years and older in seven communities of Colombia's Pacific coastal department of Chocó. Since the control activities carried out by the Chocó Sec-

Leishmaniasis: knowledge and practices in populations of the Pacific coast of Colombia tional Health Services had not had the desired results, the residents of the region were at high risk of contracting leishmaniasis, which they called bejuco (liana) and yateví. Qualitative data were collected by directly involving each community in discussion workshops and by interviewing knowledgeable informants. Using these materials as a foundation, the researchers prepared a survey with 10 closed-ended questions, which they administered to all persons over 14 years of age in each randomly chosen home visited. The results indicate that $94 \%$ of the population knew that leishmaniasis appeared as a skin disease; those not knowing that were more often women than men. With respect to the mode of transmission, 35\% of the respondents connected the disease to the bite of an insect, but they did not what the etiologic agent was and thought that the bite was inflicted by a worm that lives in the mountains. In the communities studied, the residents used a great variety of treatments to cure the disease. The treatments were based on plants, chemical substances, burning the lesions with a piece of heated metal, and, to a lesser degree, drugs. Despite being responsible for taking care of sick persons in the household, women were not acquainted with the traditional treatments used in the community. This gender difference in treatment knowledge was statistically significant, the only such statistically significant gender difference found in the research. Of the people surveyed, $45 \%$ did not know how to prevent the disease. This was more often true for women; 102 of the 155 respondents saying they did not know how to prevent the disease were women. This research emphasizes the importance of studying the knowledge and practices of local inhabitants before designing and organizing educational programs to control leishmaniasis. 